\title{
Scaling Limit of the Energy Variable for the Two- Dimensional Ising Ferromagnet
}

\author{
Joël De Coninck
}

Université de l'Etat Faculté des Sciences, B-7000-Mons, Belgium

\begin{abstract}
The critical point limit law (scaling limit) of the suitably renormalized energy variable is explicitly calculated for the two-dimensional nearestneighbour Ising cylinder with free edges. It is shown that the renormalization factor has to behave as $(2 M 2 N \ln N)^{1 / 2}$, where $2 M$ denotes the number of rows and $2 N$ the number of columns. By first taking the limit $M \rightarrow \infty$ and then $N \rightarrow \infty$, the limit law is proven to be Gaussian.
\end{abstract}

\section{Introduction}

Let us consider $d$-dimensional Ising ferromagnets with pairwise interactions. The Gibbs measure in a finite volume $\Lambda \subset \mathbb{Z}^{d}$ for a given configuration $\left\{\sigma_{A}\right\}$ at temperature $\beta^{-1}$ and external field $h$ will be taken as

$$
\begin{gathered}
d \mu_{\Lambda}\left(\beta, h ;\left\{\sigma_{\Lambda}\right\}\right)=Z_{\Lambda}^{-1}(\beta, \beta h) \exp \left\{\beta \sum_{i, j \in \Lambda} J_{i j} \sigma_{i} \sigma_{j}+\beta h \sum_{i \in \Lambda} \sigma_{i}\right\} \prod_{k \in \Lambda} d \rho\left(\sigma_{k}\right), \\
d \rho(\sigma)=\frac{1}{2}[\delta(\sigma-1)+\delta(\sigma+1)]
\end{gathered}
$$

where $J_{i j} \geqq 0$ is such that the thermodynamic limit exists and $Z_{A}\left(\beta, \beta_{h}\right)$ is the partition function normalizing the Gibbs measure. Equation (1) defines the joint probability distribution of the $|\Lambda|$ spins in the block $\Lambda$ as usual for $d$-dimensional Ising models.

Let us define the random variables magnetization $M_{A}$ and energy $E_{A}$ for a spin block $\Lambda$ by

$$
\begin{aligned}
M_{\Lambda} & =\sum_{i \in \Lambda} S_{i}, \\
E_{\Lambda} & =-\sum_{i, j \in \Lambda} J_{i j} S_{i} S_{j},
\end{aligned}
$$

where $S_{i}$ denotes the random variable associated to the $i^{t h}$ spin.

Up to this point, every concept has been introduced for one block spin $\Lambda \subset \mathbb{Z}^{d}$. One may then ask the following question: what happens if one considers block 
spin transformations like unions? This problem is not yet well formulated since one has not stipulated the objects on which the block spin transformations operate. In the probabilistic approach to the theory of critical phenomena, one is concerned with the way the block spin transformations act on probability distributions (e.g. that of $M_{A}$ or $E_{A}$ ) instead of how they act on the Hamiltonians themselves as is done in the renormalization group theory [1-10]. More precisely, one is mostly concerned with the fixed points of these transformations and their domains of attraction. This is a well defined mathematical problem since it implies that one has to find limit theorems for suitably renormalized sums of dependent random variables.

A few months ago, Newman achieved a great progress [11] for regions in the $(\beta, h)$ plane away from the critical point $\left(\beta_{c}, 0\right)$ : for models which satisfy the FKG inequalities he proved that the suitably renormalized block probability distributions for both the magnetization and energy converge weakly to a Gaussian distribution when the volume of the block tends to infinity. At the critical point however, the situation still remains unclear.

At this interesting point, some rigorous examples have been studied for the magnetization variable [12-19] and the corresponding volume variable in fluid systems [20]; but until now no such rigorous result has been obtained for the energy variable. This is precisely the aim of this paper. The interest of such kind of research has already been pointed out in, e.g. [9].

In the following, we study the critical point limit law (scaling limit) of the suitably renormalized energy variable for the two-dimensional Ising cylinder with free edges.

\section{The Two-Dimensional Ising Cylinder with Free Edges}

Consider a cylinder with $2 M$ rows and $2 N$ columns at each vertex of which a spin variable $S_{i j}$ is associated $(1 \leqq i \leqq 2 M, 1 \leqq j \leqq 2 N)$. $S_{i j}$ takes the values $\sigma_{i j}= \pm 1$ with equal probabilities. For a given configuration, the interaction energy will be taken as $(J>0)$

$$
\mathscr{E}_{2 M \times 2 N}\left(\left\{\sigma_{i j}\right\}\right)=-J \sum_{j=1}^{2 M-1} \sum_{k=1}^{2 N} \sigma_{j k} \sigma_{j+1 k}-\sum_{j=1}^{2 M} \sum_{k=1}^{2 N} \sigma_{j k} \sigma_{j k+1}
$$

with cyclic boundary conditions of the cylinder. It is well known that such a system exhibits a critical point at the thermodynamic limit when $\sinh 2 K=1$ and $h=0$. In order to study the probability distribution of the energy at this particular point, we first establish a useful (see below) connection between the moment generating function and the partition function.

Let $E_{2 M \times 2 N}$ denote the energy random variable associated to (5). Since this variable relates to the system as a whole, it is easily seen that the moment generating function of this random variable may be written, for any real $t<\beta$ :

$$
\left\langle\exp \left(t E_{2 M \times 2 N}\right)\right\rangle_{2 M \times 2 N}=Z_{2 M \times 2 N}(K-t J) / Z_{2 M \times 2 N}(K),
$$

where the mean value $\langle\cdot\rangle_{2 M \times 2 N}$ has to be taken with respect to the probability distribution of $E_{2 M \times 2 N}$ or, equivalently, with respect to the finite Gibbs state 
induced by (5); $K=\beta J$ and $Z_{2 M \times 2 N}$ denotes the partition function of the system.

Therefore, the knowledge of the partition function implies that of the moment generating function of $E_{2 M \times 2 N}$. Using the transfer matrix technique $[14,21]$, one obtains the following exact result

$$
\begin{aligned}
Z_{2 M \times 2 N}(K)= & (2 \sinh 2 K)^{2 M N}(2 \cosh K)^{-2 N} \\
& \times \prod_{\omega>0}^{<\pi}\left\{\exp \left(2 M \gamma_{\omega}\right) \cos ^{2}\left[\delta^{\prime}(\omega) / 2\right]+\exp \left(-2 M \gamma_{\omega}\right) \sin ^{2}\left[\delta^{\prime}(\omega) / 2\right]\right\}
\end{aligned}
$$

where the product has to be taken over the values $\omega=\pi(2 n-1) / 2 N$ with $n=1$, $2, \ldots, N ; \gamma_{\omega}$ is the Onsager's function which satisfies

$$
\cosh \gamma_{\omega}=\operatorname{coth} 2 K \cosh 2 K-\cos \omega,
$$

and $\delta^{\prime}(\omega)$ is defined by

$$
e^{i \delta^{\prime}(\omega)}=\left\{\frac{\left(e^{i \omega}-A\right)\left(e^{i \omega}-B\right)}{\left(e^{i \omega}-A^{-1}\right)\left(e^{i \omega}-B^{-1}\right)}\right\}^{1 / 2} \frac{1}{(A B)^{1 / 2}}
$$

with $A=\operatorname{coth} K \exp (2 K)$ and $B=\tanh K \exp (2 K)$.

For convenience, let us now introduce the following:

Lemma. For any $\omega=\pi(2 n-1) / 2 N$ with $n=1,2, \ldots, N$, one has uniformly with respect to $N$ :

$$
\left|\left(3-4 \cos \omega+\cos ^{2} \omega\right)^{-1 / 2}-\omega^{-1}\right| \leqq c^{t e} .
$$

Proof of the Lemma. The only difficulty obviously appears for small values of $\omega$. Since for such values

$$
\left(3-4 \cos \omega+\cos ^{2} \omega\right)^{1 / 2}=\omega\left(1+O\left(\omega^{2}\right)\right),
$$

one gets

$$
\left(3-4 \cos \omega+\cos ^{2} \omega\right)^{-1 / 2}-\omega^{-1}=-O(\omega) \text { as } \omega \rightarrow \text { zero. }
$$

q.e.d.

We may now formulate the result:

Theorem. For the two-dimensional nearest-neighbour Ising cylinder with free edges, the reduced variable

$$
\tilde{E}_{2 M \times 2 N}=\left(E_{2 M \times 2 N}-\left\langle E_{2 M \times 2 N}\right\rangle_{2 M \times 2 N}\right) /(2 M 2 N \ln N)^{1 / 2}
$$

at the critical point and for any real $t$, satisfies:

$$
\lim _{N \rightarrow \infty} \lim _{M \rightarrow \infty}\left\langle\exp \left(t \widetilde{E}_{2 M \times 2 N}\right)\right\rangle_{2 M \times 2 N}=\exp \left(c t^{2} / 2\right),
$$

where $c$ is some positive constant.

Proof of the Theorem. Taking the logarithm of the moment generating function of (11), one obtains the cumulant generating function which admits a Taylor's 
expansion about $t=0$ of at least order 3 for any finite $M$ and $N$. To simplify the notations, we rewrite (7) as:

$$
\ln Z_{2 M \times 2 N}(K-t J)=\ln Z_{1}(K-t J)+\ln Z_{2}(K-t J),
$$

where

and

$$
\begin{aligned}
& \ln Z_{1}(K)=2 M N \ln (2 \sinh 2 K)+2 M \sum_{\omega} \gamma_{\omega}, \\
& \ln Z_{2}(K)=-2 N \ln (2 \cosh K)+\sum_{\omega} f_{\omega},
\end{aligned}
$$

$$
f_{\omega}=\ln \left[\cos ^{2}\left(\delta^{\prime}(\omega) / 2\right)+\exp \left(-4 M \gamma_{\omega}\right) \sin ^{2}\left(\delta^{\prime}(\omega) / 2\right)\right] .
$$

Let us consider the first term in the right-hand side of (13):

$$
\begin{aligned}
\ln Z_{1}(K-t J)= & \ln Z_{1}(K)-t J\left\{4 M N \operatorname{coth}(2 K)+2 M \sum_{\omega} \gamma_{\omega}^{\prime}\right\} \\
& +(1 / 2) t^{2} J^{2}\left\{-8 M N \sinh ^{-2}(2 K)+2 M \sum_{\omega} \gamma_{\omega}^{\prime \prime}\right\}+\mathcal{O}\left(M t^{3}\right),
\end{aligned}
$$

where the prime denotes derivation with respect to $K$. Using (8), one gets

$$
\begin{aligned}
\gamma_{\omega}^{\prime} & =2 \cosh (2 K)\left[1-\sinh ^{-2}(2 K)\right] \sinh ^{-1} \gamma_{\omega} \\
\gamma_{\omega}^{\prime \prime} & =4 \sinh (2 K)\left[1-\sinh ^{-2}(2 K)\right] \sinh ^{-1} \gamma_{\omega}+8 \cosh ^{2}(2 K) \sinh ^{-3}(2 K) \sinh ^{-1} \gamma_{\omega} \\
& -2 \cosh (2 K)\left[1-\sinh ^{-2}(2 K)\right] \sinh ^{-2} \gamma_{\omega} \cosh \gamma_{\omega} \cdot \gamma_{\omega}^{\prime} .
\end{aligned}
$$

At the critical point $K=K_{c}$, one has

$$
\begin{aligned}
& \sinh \left(2 K_{c}\right)=1, \\
& \cosh \left(2 K_{c}\right)=\sqrt{2}, \\
& \cosh \gamma_{\omega}=2-\cos \omega, \\
& \sinh \gamma_{\omega}=3-4 \cos \omega+\cos ^{2} \omega .
\end{aligned}
$$

Therefore,

$$
\begin{aligned}
\ln Z_{1}\left(K_{c}-t J\right)= & \ln Z_{1}\left(K_{c}\right)+4 M N \sqrt{2} t J \\
& +(1 / 2) t^{2} J^{2}\left\{-8 M N+32 M \sum_{\omega}\left(3-4 \cos \omega+\cos ^{2} \omega\right)^{-1 / 2}\right\} \\
& +\mathcal{O}\left(M t^{3}\right) .
\end{aligned}
$$

Equivalently, this may also be written as:

$$
\begin{aligned}
& \ln Z_{1}\left(K_{c}-t J\right)-\ln Z_{1}\left(K_{c}\right)-4 M N \sqrt{2} t J \\
& =(1 / 2) t^{2} J^{2}\left\{-8 M N+32 M \sum_{\omega}\left[\left(3-4 \cos \omega+\cos ^{2} \omega\right)^{-1 / 2}-\omega^{-1}\right]\right. \\
& \left.\quad+32 M \sum_{\omega} \omega^{-1}\right\}+\mathcal{O}\left(M t^{3}\right) .
\end{aligned}
$$


Using Lemma 1 and the Schlömilch-Lemonnier inequalities [22, p. 187], it follows that the $t^{2}$-contribution grows like $4 M N \ln N$. This obviously suggests a renormalization factor of the form $(2 M 2 N \ln N)^{-1 / 2}$. With such a renormalization and by first taking the limit $M \rightarrow \infty$, one obtains

$$
\begin{aligned}
& \lim _{N \rightarrow \infty} \lim _{M \rightarrow \infty}\left\{\ln Z_{1}\left[K_{c}-t J /(2 M 2 N \ln N)^{1 / 2}\right]-\ln Z_{1}\left(K_{c}\right)-t J(4 M N / \ln N)^{1 / 2}\right\} \\
& \quad=c t^{2} J^{2},
\end{aligned}
$$

where $c$ is some positive constant. That this equality holds for any real $t$ follows from the fact that for any $t J$, there always exists an integer $M_{0}$ such that

$$
M \geqq M_{0} \Rightarrow \dot{K}_{c}-t J /(2 M 2 N \ln N)^{1 / 2}>0 .
$$

Let us now consider the second term in the right-hand side of (13). At the critical temperature and with the renormalization factor $(2 M 2 N \ln N)^{-1 / 2}$, one has

$$
\begin{aligned}
& \ln Z_{2}\left[K_{c}-t J /(2 M 2 N \ln N)^{1 / 2}\right] \\
& \quad=-\ln \left\{2 \cosh \left[K_{c}-t J /(2 M 2 N \ln N)^{1 / 2}\right]\right\}+\left.\sum_{\omega} f_{\omega}\right|_{K=K_{c}-t J /(2 M 2 N \ln N)^{12}}
\end{aligned}
$$

Using (9), $f_{\omega}$ may also be written as

$$
f_{\omega}=\ln \left\{\left[1+\exp \left(-4 M \gamma_{\omega}\right)\right]+\left[1-\exp \left(-4 M \gamma_{\omega}\right)\right] \cos \delta^{\prime}(\omega)\right\}-\ln 2,
$$

where

$$
\cos \delta^{\prime}(\omega)=[\operatorname{coth}(2 K)-\cosh (2 K) \cos \omega] / \sinh \gamma_{\omega} .
$$

For $K=K_{c}-t J /(2 M 2 N \ln N)^{1 / 2}$, by first taking the limit $M \rightarrow \infty$ :

$$
\left.\lim _{M \rightarrow \infty} \cos \delta^{\prime}(\omega)\right|_{K}=\left.\cos \delta^{\prime}(\omega)\right|_{K_{c}} .
$$

This follows easily from the continuity with respect to $K$ of $\cos \delta^{\prime}(\omega)$. Moreover, since for a fixed $\omega$ :

$$
\begin{aligned}
\left.\exp \left(\gamma_{\omega}\right)\right|_{K} & =\left.\cosh \gamma_{\omega}\right|_{K}+\left.\sinh \gamma_{\omega}\right|_{K} \geqq \inf _{0<K<+\infty}\left\{\left.\cosh \gamma_{\omega}\right|_{K}+\left.\sinh \gamma_{\omega}\right|_{K}\right\} \\
& =\left.\exp \left(\gamma_{\omega}\right)\right|_{K_{c}}
\end{aligned}
$$

one gets

$$
0 \leqq\left.\exp \left(-4 M \gamma_{\omega}\right)\right|_{K} \leqq\left.\exp \left(-4 M \gamma_{\omega}\right)\right|_{K_{c}},
$$

and, therefore,

$$
\left.\lim _{M \rightarrow \infty} \exp \left(-4 M \gamma_{\omega}\right)\right|_{K}=0
$$


Combining Eqs. (16-18), one deduces that

$$
\lim _{M \rightarrow \infty}\left\{\ln Z_{2}\left[K_{c}-t J /(2 M 2 N \ln N)^{1 / 2}\right]-\ln Z_{2}\left(K_{c}\right)\right\} 0 .
$$

This result holds also for any real $t$ since there always exists an integer $M_{0}$ such that (15) is valid.

It now suffices to consider simultaneously (14) and (19) to get the announced result (12).

$$
\text { q.e.d. }
$$

We have thus proven that, by first taking the number of rows to be infinite, the sequence of random variables $\left(\widetilde{E}_{2 M \times 2 N}\right)$ converges weakly to a Gaussian variable as $M, N \rightarrow \infty$. This shows that Newman's remark [9, following Eq. (2.45)] on the Gaussian character of the tail of the limit distribution may be extended to the whole distribution. One recovers the same limit law as in the well known central limit theorem but, due to the strong dependence of the random variables $S_{i j}$ at the critical point, one has to choose a renormalization factor of the form $(2 M 2 N \ln N)^{1 / 2}$ which is obviously stronger than the usual one for sums of independent random variables, i.e. $(2 M 2 N)^{1 / 2}$. This last factor is precisely the appropriate one away from the critical point as may be shown using the same technique.

Acknowledgements. The author is grateful to D. B. Abraham, M. Cassandro, Ph. de Gottal, G. Gallavotti and G. Jona-Lasinio for many valuable discussions. He also thanks the referee for helpful comments on a previous draft of the paper. The Fonds National de la Recherche Scientifique is also acknowledged for partial financial support.

\section{References}

1. Jona-Lasinio, G.: The renormalization group: A probabilistic view. Nuovo Cim. 26B, 99-119 (1975)

2. Jona-Lasinio, G.: Probabilistic approach to critical behavior. In: New developments in Quantum Field Theory and Statistical Mechanics, Cargèse 1976; Lévy, M., Mitter, P. (eds.) New York, London: Plenum Press 1977

3. Cassandro, M., Jona-Lasinio, G.: Asymptotic behavior of the autocovariance function and violation of strong mixing. In: Many Degrees of Freedom in Field Theory, Streit, L. (ed.). New York, London: Plenum Press 1978

4. Cassandro, M., Jona-Lasinio, G.: Critical point behaviour and probability theory. Adv. Phys. 27, 913-941 (1978)

5. Cassandro, M., Gallavotti, G. : The Lavoisier law and the critical point. Nuovo Cim. 25B, 691-705 (1975)

6. Baker, G. A., Jr., Krinsky, S.: Renormalization group structure for translationally invariant ferromagnets. J. Math. Phys. 18, 590-607 (1977)

7. Collet, P., Eckmann, J. P.: A renormalization group analysis of the hierarchical model in statistical mechanics. Berlin, Heidelberg, New York: Springer 1979

8. Sinal, Y. G.: Mathematical foundations of the renormalization group method. In: Statistical Physics in Mathematical Problems in Theoretical Physics, Dell' Antonio, G., Doplicher, S., Jona-Lasinio, G. (eds.), Lectures Notes in Physics, Vol. 80, Berlin, Heidelberg, New York: Springer 1978

9. Newman, C. M. : Critical point inequalities and scaling limits. Commun. Math. Phys. 66, 181-196 (1979) 
10. Fröhlich, J., Spencer, T.: Some recent rigorous results in the theory of phase transitions and critical phenomena. Séminaire Bourbaki No 586 (February 1982).

11. Newman, C. M.: A general central limit theorem for FKG systems. Commun. Math. Phys. 91, $75-80$ (1983)

12. Ellis, R. S., Newman, C. M.: The statistics of Curie-Weiss models. J. Stat. Phys. 19, 149-161 (1978)

13. Ellis, R. S., Newman, C. M.: Limit theorems for sums of dependent random variables occurring in statistical mechanics. Z. f. Wahrsch. Verw. Geb. 44, 117-139 (1978)

14. Abraham, D. B.: Block spins in the edge of an Ising ferromagnetic half-plane. J. Stat. Phys. 19, 553-556 (1978)

15. Aizenman, M.: Geometric analysis of $\phi^{4}$ fields and Ising models. Parts I and II. Commun. Math. Phys. 86, 1-48 (1982)

16. Fröhlich, J. : On the triviality of $\lambda \phi_{d}^{4}$ theories and the approach to the critical point in $d \gg 4$ dimensions. Nucl. Phys. B200, [FS4] 281-296 (1982)

17. Aizenman, M., Graham, R.: On the renormalized coupling constant and the susceptibility in $\phi_{4}^{4}$ field theory and the Ising model in four dimensions. Nucl. Phys. B225, [FS9] 261-288 (1983)

18. Gawedzki, K., Kupiainen, A.: Non-Gaussian fixed points of the block spin transformationhierarchical model approximation. Commun. Math. Phys. 89, 191-220 (1983) and references therein

19. Fontaine, J. R.: Scaling limit of some critical models. Commun. Math. Phys. 91, 419-430 (1983)

20. Datoussaï, H., De Coninck, J., de Gottal, Ph. : Critical point limit law for Temperley's continuous model. J. Stat. Phys. 31, 379-388 (1983)

21. Schultz, T. D., Mattis, D. C., Lieb, E. H.: Two-dimensional Ising model as a soluble problem of many fermions. Rev. Mod. Phys. 36, 856-871 (1964)

22. Mitrinovic, D. S. : Analytic inequalities. Berlin, Heidelberg, New York: Springer 1970

Communicated by J. Fröhlich

Received November 29, 1983; in revised form February 21, 1984 
